\title{
Synthesis, molecular structure, spectroscopic investigations and computational study of a potential molecular switch of 2-([1,1'-biphenyl]-4-yl)-2-methyl-6-(4-nitrophenyl)-4-phenyl-1,3 diazabicyclo [3.1.0]hex-3-ene
}

\author{
AYOUB KANAANI, DAVOOD AJLOO*, HAMZEH KIYANI*, FRESHTE SHAHERI \\ and MAJID AMIRI \\ School of Chemistry, Damghan University, Damghan, 36716-41167, Iran \\ e-mail: ajloo@du.ac.ir; hkiyani@du.ac.ir
}

MS received 29 December 2015; revised 9 April 2016; accepted 25 May 2016

\begin{abstract}
This work presents a combined experimental and theoretical study on a photochromic compound, 2-([1,1'-biphenyl]-4-yl)-2-methyl-6-(4-nitrophenyl)-4-phenyl-1,3 diazabicyclo [3.1.0]hex-3-ene, existing in closed form (' $A$ ') and open form (' $B$ '). The spectroscopic properties of the title compound have been investigated by using IR, UV-Vis and ${ }^{1} \mathrm{H}$ NMR techniques. The molecular geometry and spectroscopic data of the title compound have been calculated by using the density functional method (B3LYP) invoking 6-311G $(\mathrm{d}, \mathrm{p})$ basis set. UV-Vis spectra of the two forms were recorded. The excitation energies, oscillator strength, etc., were calculated by time-dependent density functional theory (TD-DFT). Furthermore, molecular electrostatic potential map (MEP), frontier molecular orbital analysis (HOMO-LUMO), total density of state (TDOS) and reactivity descriptors were found and discussed. We applied a first-principles computational approach to study a light-sensitive molecular switch. We find that the conductance of the two isomers varies dramatically, which suggests that this system has potential use as a molecular switch.
\end{abstract}

Keywords. Photochromism; DFT; spectroscopy; molecular switch

\section{Introduction}

Photochromism is a phenomenon associated with reversible color and absorption spectral change of a molecule. ${ }^{1}$ Photochromic compounds reversibly change geometrical structure. ${ }^{2}$ Although many types of photochromic compounds have been reported, those exhibiting photochromism in solid state are very rare. ${ }^{3}$ Depending on the special structure, 1,3diazabicyclo[3.1.0] hex-3-ene derivative crystals show different colour such as blue, purple, yellow, purple, pink, brown, etc. with UV irradiation. ${ }^{4,5}$ The photochromic behavior of " $\mathrm{A}$ " over " $\mathrm{B}$ " form can be assigned to the electron-withdrawing effect of para- $\mathrm{NO}_{2}$ substitution (scheme 1).

Heterocyclic imidazole derivatives have attracted significant consideration because of their unique optical properties and play a main role in chemistry as an intermediary for synthetic reactions. ${ }^{6}$ Imidazole nucleus forms the primary structure of several wellknown biomolecules and also has considerable analytical applications by virtue of their chemiluminescence and fluorescence. ${ }^{7}$ Aromatic nitro compounds and their

*For correspondence derivatives are used as analytical reagents, solvents, and are important intermediates in the organic synthesis of explosives, pesticides, drugs, and perfumes. ${ }^{8,9}$

With continued shrinking of the size of conventional silicon-based electronics, molecular electronics has been proposed to be the future nanoscale electronic devices. ${ }^{10}$ Recently, many potentially applicable molecular electronic devices, like molecular switches, fieldeffect transistors, wires and rectifiers devices have been investigated. ${ }^{11}$ A wide range of molecular switches has been presented in the literature, activated with different kinds of external motive. ${ }^{12}$ Light is a marvelous stimulus because its fast response time. In the present work, Non-equilibrium Green's function (NEGF) formalism combined with first-principles density functional theory (DFT) were used to investigate molecular conductance of the title compound.

$A b$ initio quantum-mechanical method is at present widely used for simulating IR spectrum and studying isomeric compounds. ${ }^{13-17}$ In this study, vibrational spectra, optimized geometry, electronic absorption spectra, reactivity descriptors, molecular electrostatic potential and the electron transport properties of the title compound have been studied. 


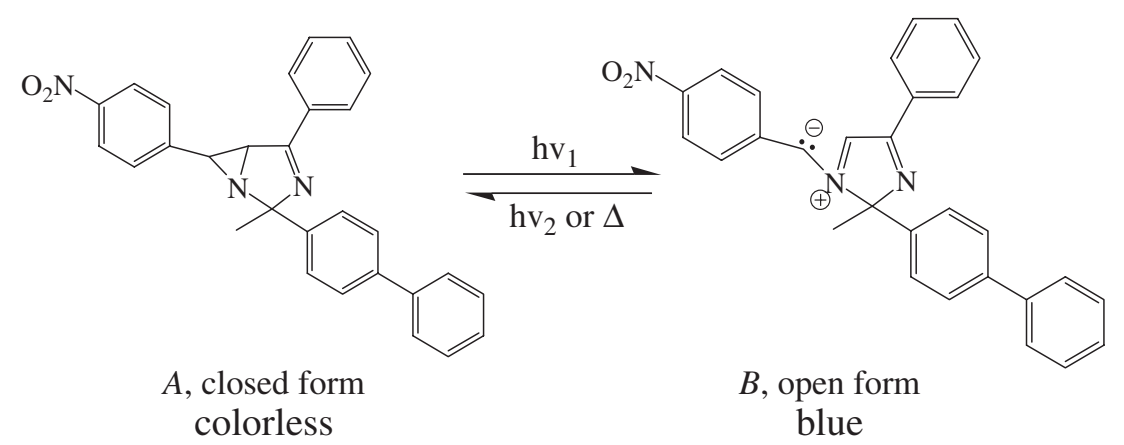

Scheme 1. Structures and Photochromic reaction of the title compound.

\section{Experimental}

\subsection{Materials and measurements}

The mid-IR spectra were obtained in the 4000$400 \mathrm{~cm}^{-1}$ region with a spectral resolution of $2 \mathrm{~cm}^{-1}$ by averaging the results of 10 scans with a PerkinElmer RXI Fourier Transform spectrophotometer using $\mathrm{KBr}$ pellet technique. The ultraviolet absorption spectra were recorded in the range $200-800 \mathrm{~nm}$ using Perkin-Elmer lambda 25 recording spectrophotometer equipped with a $10 \mathrm{~mm}$ quartz cell. The photoinduced form was formed upon UV irradiation (Hg lamp DRSh-260+ UV-transmitting glass filters). The NMR spectrum was recorded for the title compound at the ambient temperature on a Brucker AVANCE DRX $400 \mathrm{MHz}$ using $\mathrm{CDCl}_{3}$ as a solvent. All the chemicals and reagents were purchased from Merck and Fluka and were used without further purification. The development of the reaction was checked by thin layer chromatography (TLC) analysis on silica gel $60 \mathrm{GF}_{254}$ aluminum sheets, using ethyl acetate: petroleum ether (1: 3 ) as the mobile phase. The spots were exposed to UV light and iodine vapor.

Synthesis of the 2-([1,1'-biphenyl]-4-yl)-2-methyl-6(4-nitrophenyl)-4-phenyl-1,3 diazabicyclo [3.1.0]hex3-ene is explained in Supplementary Information.

\subsection{Computational methods}

The calculations for the title compound have been done with a hybrid functional B3LYP at $6-311 \mathrm{G}(\mathrm{d}, \mathrm{p})$ and basis set. All the calculations were performed using G03 program package. ${ }^{18}$ The molecular structure of the title compound in the ground state was optimized. For all the calculations for this study, optimized structural parameters were used. The vibrational frequencies were calculated and scaled. ${ }^{19}$ The Lorentzian function has been employed for deconvolution of IR spectrum using the Genplot package. ${ }^{1} \mathrm{H}$ NMR chemical shifts were calculated using the standard GIAO/DFT (GaugeIndependent Atomic Orbital). ${ }^{20}$ Electronic excitations of the title compound were calculated by using TD-DFT in the gas phase. ${ }^{21}$

The transport properties of the molecular junction were calculated using the TranSIESTA package ${ }^{22}$ which is based on the combination of DFT with the nonequilibrium Green's function (NEGF) technique. In NEGF theory, the molecular wire junction is separated into three regions: left electrode (L), contact region (C), and right electrode $(\mathrm{R})$. In the lateral dimension, a $6 \times 6$ supercell was used, large enough to remove interactions between periodic images. Based on previous issues about self-assembled monolayers (SAMs), it is accepted that hydrogen atoms are dissociated upon adsorption to metal surfaces. ${ }^{23}$ So, the two terminal hydrogen atoms bonded to the sulfur atoms are omitted from the optimized structure, and the remaining segment is put between two parallel $\mathrm{Au}(111)$ surfaces, which have a face-centered cubic (f.c.c.) lattice.

The structural optimization is accomplished in the SIESTA package ${ }^{24}$ with the maximum force of $0.02 \mathrm{eV} / \AA$, and core electrons are modeled using Troullier-Martins ${ }^{25}$ soft norm-conserving pseudopotentials. The Perdew-Burke-Ernzerhof (PBE) exchangecorrelation functional was adopted for the generated gradient approximation (GGA) ${ }^{26}$ There is a good compromise of prior theoretical simulations with experimental measurements. ${ }^{27}$ The Au atoms are described as a single- $\xi$ plus single polarization (SZP) basis set while for the other atoms a double- $\xi$ plus single polarization (DZP) basis set is used. The current through the device is computed using the Landauer-Büttiker formula in the TranSIESTA package. ${ }^{22}$

$$
I=\frac{2 e}{h} \int T(E, V)\left[f\left(E-\mu_{\mathrm{L}}\right)-f\left(E-\mu_{\mathrm{R}}\right)\right] d E
$$

Here, $e$ is the electron charge, $h$ Planck's constant, $T(E, V)$ the transmission function at energy $E$ under bias voltage $V$, and $f\left(E-\mu_{L(R)}\right)$ is the Fermi-Dirac 
distribution function with the electrochemical potential $\mu_{L(R)}$ of the left (right) electrode.

\section{Results and Discussion}

\subsection{Geometrical parameters}

The optimized geometrical parameters of the title compound were calculated by using DFT/B3LYP method with $6-311 \mathrm{G}(\mathrm{d}, \mathrm{p})$ basis set which are listed in table $\mathrm{S} 1$ in accordance with numbering scheme given in figure 1 . Some selected geometric parameters $\left(\AA,^{\circ}\right)$ of " $\mathrm{A}$ " and " $\mathrm{B}$ " forms are presented in table 1 . The optimized structure may be contrasted with other similar systems for which the crystal structures have been solved. Since the identification of all the normal modes of vibration of large molecules is not explicit, we tried to streamline the problem by considering each molecule as substituted benzene as this idea has been used several times in the past. ${ }^{28}$ In the following discussion,
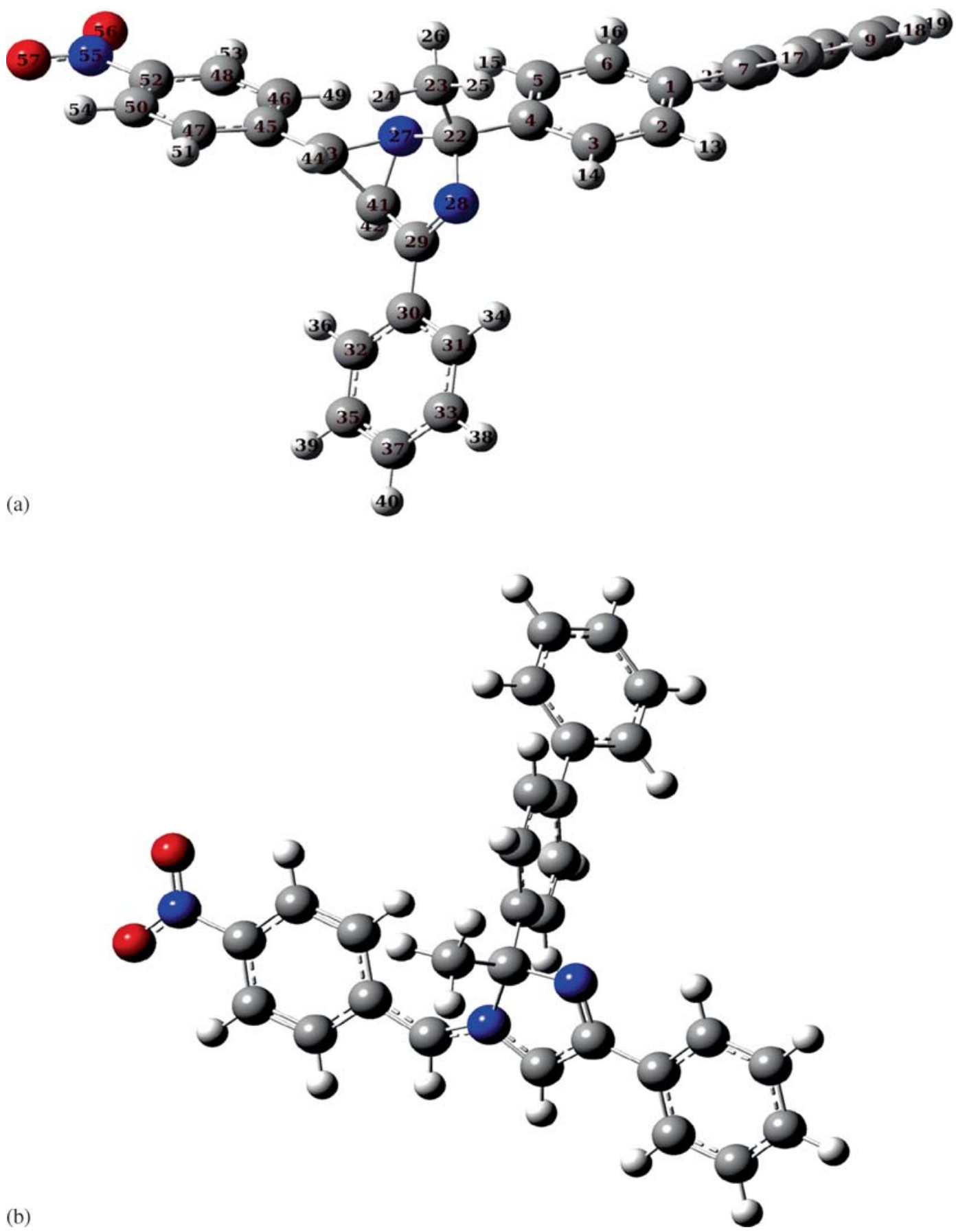

(b)

Figure 1. Optimized geometry by B3LYP/6-311++G(d,p) for (a) "A" and (b) "B" forms. 
Table 1. Selected geometric parameters of "A" and "B" forms, atom labeling according to figure 1.

\begin{tabular}{|c|c|c|c|c|c|}
\hline Parameters & "A" & "B" & & "A" & “B” \\
\hline Bond lengths $(\AA ̊)$ & & & Bond lengths $(\AA)$ & & \\
\hline $\mathrm{C}_{4}-\mathrm{C}_{22}$ & 1.5347 & 1.5319 & $\mathrm{~N}_{27}-\mathrm{C}_{41}$ & 1.4612 & 1.0790 \\
\hline $\mathrm{C}_{22}-\mathrm{C}_{23}$ & 1.5361 & 1.5361 & $\mathrm{C}_{41}-\mathrm{H}_{42}$ & 1.0870 & 1.3530 \\
\hline $\mathrm{C}_{23}-\mathrm{H}_{24}$ & 1.0933 & 1.0923 & $\mathrm{~N}_{27}-\mathrm{C}_{43}$ & 1.4665 & 1.3465 \\
\hline $\mathrm{C}_{22}-\mathrm{N}_{28}$ & 1.4759 & 1.3079 & $\mathrm{C}_{43}-\mathrm{H}_{44}$ & 1.0888 & 1.0858 \\
\hline $\mathrm{N}_{28}-\mathrm{C}_{29}$ & 1.2850 & 1.4767 & $\mathrm{C}_{43}-\mathrm{C}_{45}$ & 1.4899 & 1.4349 \\
\hline $\mathrm{C}_{29}-\mathrm{C}_{30}$ & 1.4721 & 1.4027 & & & \\
\hline Bond angles $\left({ }^{\circ}\right)$ & & & Bond angles $\left({ }^{\circ}\right)$ & & \\
\hline $\mathrm{A}(4,22,23)$ & 109.6 & 116.7 & $\mathrm{~A}(22,27,41)$ & 105.0 & 105.3 \\
\hline $\mathrm{A}(22,23,24)$ & 111.7 & 108.5 & $\mathrm{~A}(27,41,42)$ & 119.3 & 121.3 \\
\hline $\mathrm{A}(22,23,25)$ & 109.4 & 109.3 & $\mathrm{~A}(22,27,43)$ & 116.0 & 130.6 \\
\hline $\mathrm{A}(22,23,26)$ & 109.9 & 112.6 & $\mathrm{~A}(27,43,44)$ & 118.4 & 111.5 \\
\hline $\mathrm{A}(4,22,27)$ & 108.7 & 110.7 & $\mathrm{~A}(27,43,45)$ & 117.6 & 134.0 \\
\hline $\mathrm{A}(4,22,28)$ & 109.0 & 107.9 & $\mathrm{~A}(43,45,46)$ & 121.5 & 128.6 \\
\hline $\mathrm{A}(22,28,29)$ & 109.2 & 108.0 & $\mathrm{~A}(43,45,47)$ & 119.4 & 114.8 \\
\hline $\mathrm{A}(28,29,30)$ & 123.7 & 122.1 & & & \\
\hline Dihedral angles $\left({ }^{\circ}\right)$ & & & Dihedral angles $\left({ }^{\circ}\right)$ & & \\
\hline $\mathrm{D}(3,4,22,23)$ & 84.8 & 35.0 & $\mathrm{D}(28,29,30,31)$ & -9.8 & -6.0 \\
\hline $\mathrm{D}(4,22,23,24)$ & -176.8 & -179.6 & $\mathrm{D}(28,29,30,32)$ & 169.8 & -179.9 \\
\hline $\mathrm{D}(4,22,23,25)$ & -57.1 & -62.5 & $\mathrm{D}(4,22,27,41)$ & 111.3 & 115.2 \\
\hline $\mathrm{D}(4,22,23,26)$ & 62.0 & 59.5 & $\mathrm{D}(4,22,27,43)$ & 177.2 & -68.3 \\
\hline $\mathrm{D}(3,4,22,27)$ & -153.0 & -87.5 & $\mathrm{D}(22,27,43,44)$ & 8.8 & -173.1 \\
\hline $\mathrm{D}(3,4,22,28)$ & -36.4 & -87.5 & $\mathrm{D}(22,27,43,45)$ & 153.3 & 4.7 \\
\hline $\mathrm{D}(4,22,28,29)$ & -110.8 & -117.4 & $\mathrm{D}(27,43,45,46)$ & 14.5 & 15.9 \\
\hline $\mathrm{D}(22,28,29,30)$ & 176.8 & 179.3 & $\mathrm{D}(27,43,45,47)$ & -166.8 & -166.1 \\
\hline
\end{tabular}

the nitrobenzene, benzene, and biphenyl are assigned as PhI, PhII and PhIII, respectively, and the 4-methyl-3,5diaza-bicycle [3.1.0]hex-2-ene as Ring. For "B" form, $\mathrm{PhI}, \mathrm{PhII}$ and PhIII are similar and 1,2-dimethyl-2,5dihydro-1H-imidazole is assigned Ring.

Dhanabal et al. ${ }^{29}$ reported the $\mathrm{N}-\mathrm{O}$ and $\mathrm{C}-\mathrm{N}$ bond lengths in the range of $1.198-1.238$ and $1.440-1.525 \AA$. Sundaraganesan et al., ${ }^{30}$ stated $\mathrm{C}-\mathrm{N}$ and $\mathrm{N}-\mathrm{O}$ bond lengths as $1.453,1.460 \AA$ and in the range 1.273 $1.275 \AA$, respectively. For " $\mathrm{A}$ " form, the N-O bond lengths are $1.231 \AA$ and $\mathrm{C}-\mathrm{N}$ bond lengths are 1.461$1.502 \AA$, and for "B" form, N-O and $\mathrm{C}-\mathrm{N}$ bond lengths are in the range 1.236-1.236 $\AA$ and $1.347-$ $1.553 \AA$, respectively, which are in agreement with the reported values. The $\mathrm{CNO}$ angles are reported ${ }^{33}$ in the range $117.4-118.7^{\circ}$, whereas for the " $\mathrm{A}$ " form it is $117.7^{\circ}$ and for " $\mathrm{B}$ " form it is $117.9-118.0^{\circ}$. Sridevi et al. ${ }^{31}$ reported $\mathrm{N}=\mathrm{C}, \mathrm{N}_{28}-\mathrm{C}_{22}$ bond lengths and $\mathrm{O}-\mathrm{N}-\mathrm{O}, \mathrm{O}-\mathrm{N}-\mathrm{C}$ bond angles as $1.283,1.357 \AA$ and $125.3,118.0-116.6^{\circ}$ for 5-chloro-1-methyl-4-nitro$1 \mathrm{H}$-imidazole. In the present case, for " $\mathrm{A}$ " form the respective bond lengths are $1.285,1.476 \AA$ and bond angles 124.6, 117.7, and for "B" form bond lengths are 1.308, $1.448 \AA$ and bond angles are 124.2, $117.9-118.0^{\circ}$.
The Global minimum energies obtained for dimer were -2867.13672 and -2867.11578 Hartrees by DFT method and the corrected intermolecular energies were -2867.13486 and -2867.11399 Hartrees in BSSE method for "A" and "B" forms, respectively. The energy differences were only 0.0018621 and 0.0017914 Hartrees confirming that in the present case this method seemed to be compatible.

\subsection{Vibrational analysis}

The title compound contains 57 atoms, and it has 165 normal modes of vibration. The assignments of the observed infrared vibrational bands have been performed and compared with the calculated frequencies. The calculated wavenumbers are generally scaled by scaling factor to evaluate with observed wavenumbers. ${ }^{15}$ The experimental FT-IR spectrum of the title compound in the region $4000-400 \mathrm{~cm}^{-1}$ is shown in figure 2. For obvious identification of these bands, we used band deconvolution techniques in the IR spectrum. A deconvoluted IR spectrum of the title compound is shown in figure S1 (Supplementary Information). The observed IR bands and calculated wavenumbers (scaled) and assignments are given in table S2 


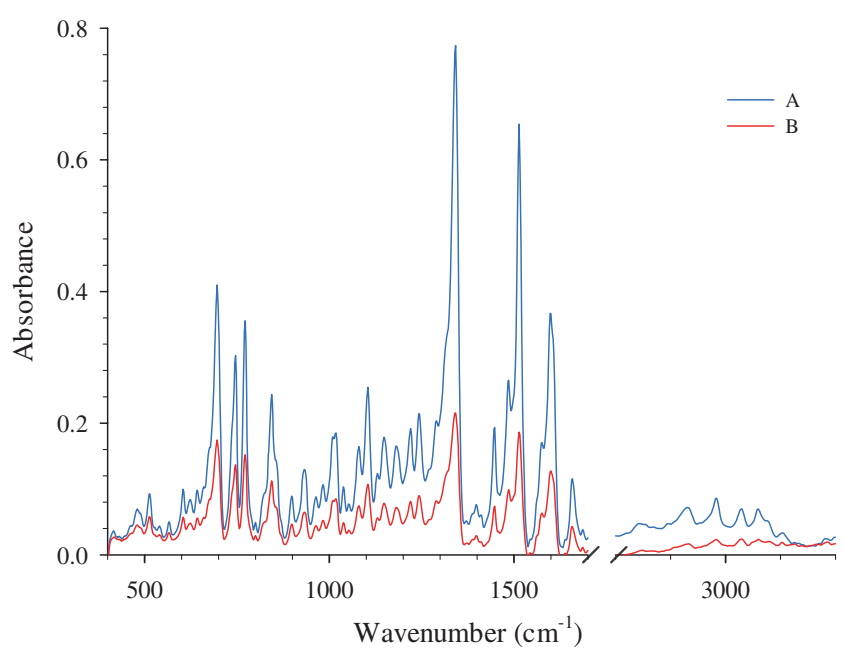

Figure 2. FT-IR spectra (experimental) for "A", before irradiation; and "B", after irradiation for $50 \mathrm{~s}$ at $300 \mathrm{~nm}$.

(Supplementary Information). The correlation diagram for the calculated and the experimental frequencies of "A" and "B" forms are shown in figure S2 (Supplementary Information).

The connection between the calculated and experimental wavenumbers is linear and described by the following equations:

$$
\begin{array}{rr}
\text { For "A" form: } \quad v_{\text {cal. }}=0.9864 v_{\text {exp. }}+8.4408 \\
\left(\mathrm{R}^{2}=0.9978\right) \\
\text { For "B" form: } \quad v_{\text {cal. }}=0.9874 v_{\text {exp. }}+0.5383 \\
\left(\mathrm{R}^{2}=0.9983\right)
\end{array}
$$

Aromatic nitro compounds displayed strong absorption due to the asymmetric and symmetric vibrations of the $\mathrm{NO}_{2}$ group in $1570-1485 \mathrm{~cm}^{-1}$ and 1370 $1320 \mathrm{~cm}^{-1}$, respectively. ${ }^{32}$ Deconvoluted IR spectra of "A" form showed the presence of two bands observed to the asymmetric vibrations of the $\mathrm{NO}_{2}$ group in 1602, $1498 \mathrm{~cm}^{-1}$ (see figure S1a in Supplementary Information) and in $1603,1552 \mathrm{~cm}^{-1}$ (B3LYP). The symmetric stretching mode for "A" form was observed at $1348 \mathrm{~cm}^{-1}$ in FT-IR which coincides with the theoretically computed value $1342 \mathrm{~cm}^{-1}$.

The $\mathrm{NO}_{2}$ scissors ${ }^{33}$ appear at higher wavenumbers $\left(850 \pm 60 \mathrm{~cm}^{-1}\right)$ when conjugated to $\mathrm{C}-\mathrm{C}$ or aromatic molecules. For nitrobenzene, $\delta \mathrm{NO}_{2}$ is reported ${ }^{26}$ at $852 \mathrm{~cm}^{-1}$. Deconvoluted IR spectra for "A" form indicated the presence of two bands at 852 and $774 \mathrm{~cm}^{-1}$ in the IR spectrum and 848 and $819 \mathrm{~cm}^{-1}$ in B3LYP are assigned as $\delta \mathrm{NO}_{2}$ modes. In aromatic compounds, the wagging mode $\omega \mathrm{NO}_{2}$ is expected in the region $740 \pm$ $50 \mathrm{~cm}^{-1}$ with a moderate to strong intensity, a region in which $\gamma \mathrm{CH}$ is also active. ${ }^{26}$ Deconvoluted IR spectra for " $\mathrm{A}$ " form indicates the presence of two bands at 738 and $644 \mathrm{~cm}^{-1}$ in the IR spectrum, and 725 and $680 \mathrm{~cm}^{-1}$ in B3LYP calculation that belong to $\gamma \mathrm{NO}_{2}$ modes. The rocking mode, $\rho \mathrm{NO}_{2}$ is active in the region $540 \pm$ $70 \mathrm{~cm}^{-1}$ in aromatic nitro compounds. ${ }^{26}$ For "A" form, the FT-IR band observed at $512 \mathrm{~cm}^{-1}$ corresponds to $\rho \mathrm{NO}_{2}$ rocking vibration while theoretically computed value was $516 \mathrm{~cm}^{-1}$. Suryanarayana et al., ${ }^{34}$ reported the range $65 \pm 10 \mathrm{~cm}^{-1}$ as the $\tau \mathrm{NO}_{2}$ for aromatic compounds. In the " $\mathrm{A}$ " form, the deformation modes of $\mathrm{NO}_{2}$ are assigned at 65 and $27 \mathrm{~cm}^{-1}$ (B3LYP).

The $\mathrm{C}=\mathrm{N}$ stretching skeletal bands are expected in the $1585 \mathrm{~cm}^{-1} .35$ Deconvoluted IR spectra of "A" form showed the appearance of two bands in 1664, $1578 \mathrm{~cm}^{-1}$ in IR spectrum. The identification of the $\mathrm{C}-$ $\mathrm{N}$ vibration is a very difficult task since mixing of several bands is possible in this region. Melardi et al., ${ }^{36}$ assigned the $\mathrm{C}-\mathrm{N}$ stretching absorption in $1305 \mathrm{~cm}^{-1}$ for aromatic amines. In "A" form, the theoretical calculation gives 1364,1324 and $1231 \mathrm{~cm}^{-1}$ as C-N stretching modes matching with bands at 1398, 1334 and $1224 \mathrm{~cm}^{-1}$ in the IR spectrum.

The band at $2739 \mathrm{~cm}^{-1}$ is assigned to the $\mathrm{CH}_{3}$ asymmetric stretching. ${ }^{37}$ The $\mathrm{C}-\mathrm{H}$ stretching in methyl group occurs at lower frequencies than the use of the aromatic ring (3100-3000 $\left.\mathrm{cm}^{-1}\right)$. The B3LYP calculations give $v_{\mathrm{a}} \mathrm{CH}_{3}$ at 3031,3022 and $3015 \mathrm{~cm}^{-1}$ and $v_{\mathrm{s}} \mathrm{CH}_{3}$ at $2944 \mathrm{~cm}^{-1}$. The bands at 2895 and $2845 \mathrm{~cm}^{-1}$ in IR spectrum are assigned as $v_{\mathrm{a}} \mathrm{CH}_{3}$ and $v_{\mathrm{s}} \mathrm{CH}_{3}$ for the "A" form. For the "A" form, the scissoring mode of the $\mathrm{CH}_{3}$ group is assigned at 1449,1440, 1364 and $1360 \mathrm{~cm}^{-1}$ (B3LYP) and 1446, $1398 \mathrm{~cm}^{-1}$ (IR). In "A" form, the $\mathrm{CH}_{3}$ wagging mode is observed at $1110,1072 \mathrm{~cm}^{-1}$ and in the IR spectrum at 1212, 1120, 1110, 1088 and $1062 \mathrm{~cm}^{-1}$ theoretically. The rocking vibrations of the $\mathrm{CH}_{3}$ group are generally observed in the region 1070 $1010 \mathrm{~cm}^{-1} .37$ The band at $926 \mathrm{~cm}^{-1}$ in the IR spectrum and at 918, 908, 882 and $871 \mathrm{~cm}^{-1}(\mathrm{~B} 3 \mathrm{LYP})$ are assigned as $\rho \mathrm{CH}_{3}$ mode for the "A" form. The bands at 259, 238, 221 and $206 \mathrm{~cm}^{-1}$ (B3LYP) are assigned as the twisting mode $\tau \mathrm{CH}_{3}$.

Sajan et al.,${ }^{38}$ assigned the $\mathrm{C}-\mathrm{H}$ stretching vibrations absorption in the region $3064-3012 \mathrm{~cm}^{-1}$ for aromatic amines. In " $\mathrm{A}$ " form the bands observed at 3182, 3103, $3079,3060,3028,2982$ and $2929 \mathrm{~cm}^{-1}$ in the IR spectrum are assigned to the $\mathrm{C}-\mathrm{H}$ stretching modes of the phenyl rings. The B3LYP calculations give these modes in the range $3048-3086 \mathrm{~cm}^{-1}$.

The $\mathrm{C}-\mathrm{H}$ out-of-plane deformations of $\gamma \mathrm{CH}$ is observed between 1000 and $700 \mathrm{~cm}^{-1}$.33,34 The aromatic $\mathrm{C}-\mathrm{H}$ in-plane bending modes of benzene and its derivatives are observed in the region $1300-1000 \mathrm{~cm}^{-1}$. The $\mathrm{C}-\mathrm{H}$ out-of-plane bending modes, usually of medium intensity, arise in the region $950-600 \mathrm{~cm}^{-1} .{ }^{39}$ 
In the case of "A" form, the bands observed at 1334 , $1244,1180,1142,1096,1012 \mathrm{~cm}^{-1}$ are assigned to the $\mathrm{C}-\mathrm{H}$ in-plane bending modes of the phenyl rings. The aromatic $\mathrm{C}-\mathrm{H}$ out-of-plane bending vibrations of the title compound is assigned to the medium to weak bands observed at $926,754,692,600,560 \mathrm{~cm}^{-1}$ for "A" form in the infrared spectrum.

The IR frequency regions for the " $\mathrm{B}$ " form are similar to "A" form.

\section{$3.3{ }^{1}$ H NMR spectrum}

The ${ }^{1} \mathrm{H}$ spectrum of the title compound in "A" form was recorded in $\mathrm{CDCl}_{3}$ (figure 3). The ${ }^{1} \mathrm{H}$ NMR theoretical chemical shifts and the assignments of the title compound are presented in table S3 (Supplementary Information), according to atom numbering in the figure 1 . The geometry of the title compound, together with that of tetramethylsilane (TMS), is fully optimized. Then, ${ }^{1} \mathrm{H}$ chemical shift calculation of the compound was done by the same method using 6-311G(d,p) basis set IEFPCM/chloroform solution. ${ }^{20}$ The chemical shifts of the aliphatic proton appeared in the high field while aromatic protons of organic compounds are usually seen in the range of $7.00-8.00 \mathrm{ppm}$. The signals of the eighteen aromatic protons $\left({ }^{1} \mathrm{H}\right)$ of the title compound were calculated theoretically as 7.37-8.61 ppm and experimentally observed at $7.27-8.28 \mathrm{ppm}$. We predicted $\mathrm{H}_{19}$ at $7.37 \mathrm{ppm}$ in the lower field and $\mathrm{H}_{54}$ at $8.61 \mathrm{ppm}$ in the highest field of the aromatic region. It could be seen from table S3 that chemical shifts that is obtained through theory are in agreement with the experimental NMR data.

\subsection{Molecular electrostatic potential (MEP) surface}

Molecular electrostatic potential (MEP) is used for comparative reactivities towards electrophilic attack and nucleophilic reactions. ${ }^{40}$ To predict reactive sites for electrophilic and nucleophilic attack for "A" and "B" forms, the MEP at the B3LYP/6-311G(d,p) method was calculated. The negative (red and yellow) and the positive (blue) regions of MEP were related to electrophilic and nucleophilic reactivities are shown in figure S3 (Supplementary Information) where blue shows the strongest attraction and red shows the strongest repulsion. MEP color scale is such that $\delta^{+} \rightarrow \delta^{-}$in the direction red $\rightarrow$ blue. The maximum negative electrostatic potential is prominent over $\mathrm{O}_{56}$ and $\mathrm{O}_{57}$ atoms. The maximum negative electrostatic potential value for these electrophilic sites calculated at B3LYP/6-311G $(\mathrm{d}, \mathrm{p})$ are about $-22.339\left(\mathrm{O}_{56}\right)$, $-22.340\left(\mathrm{O}_{56}\right)$ and $-22.371\left(\mathrm{O}_{56}\right),-22.371\left(\mathrm{O}_{56}\right)$ a.u. for "A" and "B" forms. The fitting point charges to those electrostatic potentials are $-0.443\left(\mathrm{O}_{56}\right),-0.451\left(\mathrm{O}_{57}\right)$ and $-0.437\left(\mathrm{O}_{56}\right),-0.440\left(\mathrm{O}_{57}\right)$. For the feasible nucleophilic reaction, the maximum positive region is located on hydrogen bond to $\mathrm{C}$ atoms. The higher
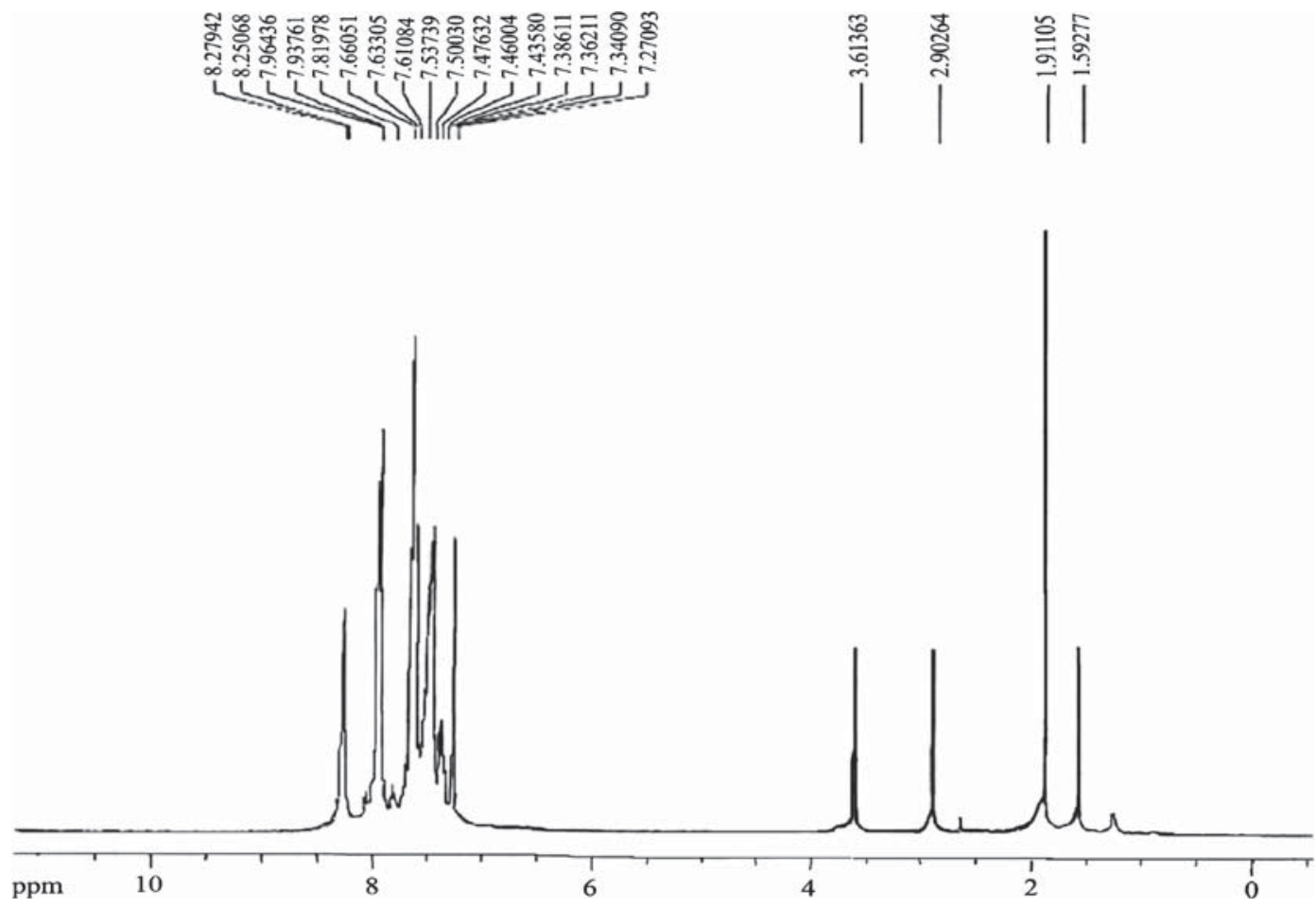

Figure 3. ${ }^{1} \mathrm{H} \mathrm{NMR}\left(400.224 \mathrm{MHz}, \mathrm{CDCl}_{3}\right)$ spectrum of "A" form at $294.8 \mathrm{~K}$. 
electronegativity in the nitro group makes it the most reactive part of the molecule.

\subsection{HOMO-LUMO band gap}

Gauss-Sum 2.2 Program ${ }^{41}$ was used to calculate group contributions to the molecular orbitals (HOMO and LUMO) and provide the density of the state (DOS) as shown in figure S4 (Supplementary Information). DOS plot shows population analysis per orbital and indicates an easy outlook of the makeup of the molecular orbitals in a certain energy range. The energy split between the HOMOs and LUMOs are the critical parameters in special molecular electrical transport properties which help in the measure of electron conductivity. ${ }^{42}$ The HOMO represents the ability to donate an electron whereas LUMO represents the ability to obtain the electron. The energy difference between the HOMO and LUMO illustrate the charge conduction interaction within the molecule The frontier orbital gaps in the case of "A" and "B" forms were found to be $3.592 \mathrm{eV}$ and $2.397 \mathrm{eV}$, respectively, obtained by TD-DFT method using 6-311G(d,p) basis set in the gas phase (see figure S5 in Supplementary Information). The conjugated molecules are noted for low HOMO-LUMO separation. Therefore, an electron density conduction happens from the maximum aromatic region of the $\pi$-conjugated system in the electron-donor side to its electron-withdrawing part. Upon excitation, intramolecular electron displacement occurs from the phenyl group to the nitrobenzene moiety, where phenyl and nitrobenzene groups acted as electron donor and acceptor, respectively.

\subsection{Electronic spectra}

Experimentally observed UV-Vis absorption spectra of "A" and "B" forms recorded in ethanol solvent are presented in figure 4.

The first excited state of open form has been fully optimized based on the optimized ground state geometric conformation as the initial conformation with the TDDFT method..$^{21}$ The calculated absorption spectra in this work are for the first 10 singlet excited states and all the calculated emission spectra are for the first 6 low lying singlet excited states. Based on the TDDFT/B3LYP/6-311G(d,p) calculated level, the corresponding absorption and fluorescence spectra of open form are displayed in figure S6 (Supplementary Information). It should be noted that the strong absorption peak for open form is located at $430 \mathrm{~nm}\left(23256 \mathrm{~cm}^{-1}\right)$ (listed in table 2), which is in

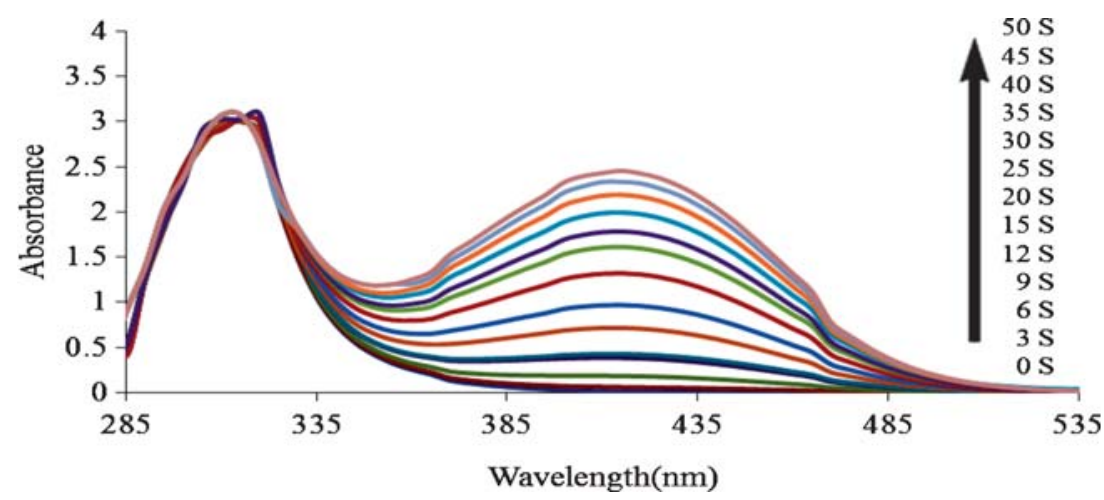

Figure 4. UV-Vis absorption spectra of the title compound upon irradiation under $300 \mathrm{~nm}$ light in $\mathrm{EtOH}$ at a concentration of $2 \times 10^{-3} \mathrm{M}$ with a $10 \mathrm{~mm}$ quartz cell before and after successive UV irradiation $\left(\lambda_{\max }=310 \mathrm{~nm}\right.$ for " $\mathrm{A}$ " and $310,415 \mathrm{~nm}$ for “ $\mathrm{B}$ ”).

Table 2. Experimental and calculated absorption wavelength and oscillator strengths of "A" and "B" forms using the TDDFT method at the B3LYP/6-311G(d,p) level.

\begin{tabular}{|c|c|c|c|c|c|c|c|}
\hline \multirow[b]{2}{*}{ Comp. } & \multicolumn{2}{|c|}{ Ethanol } & \multicolumn{2}{|c|}{ Gas } & \multirow{2}{*}{$\begin{array}{c}\text { Exp. } \\
\lambda(\mathrm{nm})\end{array}$} & \multirow[b]{2}{*}{ Assignment } & \multirow[b]{2}{*}{${ }^{\mathrm{a}}$ Gas major contribution } \\
\hline & $\overline{\lambda(\mathrm{nm})}$ & $f$ & $\overline{\lambda(\mathrm{nm})}$ & $f$ & & & \\
\hline “A" & 310.3 & 0.085 & 318.6 & 0.136 & 310 & $\pi \rightarrow \pi^{*}$ & $\begin{aligned} \mathrm{H} & \rightarrow \mathrm{L}(75 \%), \\
\mathrm{H}-2 & \rightarrow \mathrm{L}+1(21 \%)\end{aligned}$ \\
\hline "B" & $\begin{array}{l}315.8 \\
430.2\end{array}$ & $\begin{array}{l}0.070 \\
0.848\end{array}$ & $\begin{array}{l}318.4 \\
443.8\end{array}$ & $\begin{array}{l}0.089 \\
0.663\end{array}$ & $\begin{array}{l}310 \\
\mathbf{4 1 5} \\
\end{array}$ & $\begin{array}{c}\pi \rightarrow \pi^{*} \\
\mathrm{n} \rightarrow \pi^{*}\end{array}$ & $\begin{array}{c}\mathrm{H}-1 \rightarrow \mathrm{L}(91 \%) \\
\mathrm{H} \rightarrow \mathrm{L}(98 \%)\end{array}$ \\
\hline
\end{tabular}

${ }^{\mathrm{a}} \mathrm{H}: \mathrm{HOMO}, \mathrm{L}: \mathrm{LUMO}$ 
consistent with $24096 \mathrm{~cm}^{-1}$ (415 nm) in the experiment. The results of open form shown in figure S6 reveal a normal Stokes shifted emission maximum at $546 \mathrm{~nm}$ $\left(18315 \mathrm{~cm}^{-1}\right)$ in ethanol.

For both "A" and "B" forms of the title compound, wavelength $(\lambda)$, oscillator strength $(f)$ and major contributions of calculated transitions are given in table 2 . The calculated excitation energies of the transition are compared with the experimental values and the results are in good agreement. ${ }^{15}$

The UV-Vis band in "A" form is observed at $310 \mathrm{~nm}$ (colorless), and for " $\mathrm{B}$ " form bands are observed at 310 , $415 \mathrm{~nm}$ (blue color) due to the formation of zwitterionic doubly charged imine ylide (open form). The observed band at $310 \mathrm{~nm}$ is due to the $\pi \rightarrow \pi^{*}$ transition. The more important band observed at $415 \mathrm{~nm}$ for " $B$ " form belong to the dipole-allowed $n \rightarrow \pi^{*}$ transition. "B" form was also converted to "A" form after being held at $90^{\circ} \mathrm{C}$ for $4-5 \mathrm{~min}$.

The results of TD-DFT show an appreciable redshift in solvent and the degree of red-shift in vacuum is more significant than that in solvent. The discrepancy between vacuum and solvent effects in TD-DFT calculations may result from two aspects. The first aspect is that smaller gap of materials which induces smaller excited energies. The other is solvent effects. Experimental measurements of electronic absorption are usually performed in solution. Solvent, especially in polar solvent, could affect the geometry and electronic structure as well as the properties of molecules through the long-range interaction between solute and solvent molecules.

The HOMO-LUMO gap of the title compound in ethanol (3.659 and $2.552 \mathrm{eV}$ for "A" and "B" forms) at TD-DFT/B3LYP/6-311G(d,p) theory level is bigger than that in vacuum (3.5918 and $2.3962 \mathrm{eV}$ for " $\mathrm{A}$ " and " $\mathrm{B}$ " forms). A general hypochromic shift (blue shift) was noticed from the gas phase to the polar solvent ethanol. We propose that these observations are due to the role of the nitrogen dioxide $\left(\mathrm{NO}_{2}\right)$ functionality which induces charge- transfer and intermolecular hydrogen bonding. The observed blue shift can be described by the hydrogen acceptor ability of the organic molecule and the hydrogen donor ability of the relevant solvents. ${ }^{43}$ This fact designates that the solvent effects destabilize the frontier orbitals of the title compound. Hence, it induces blue-shift of the absorption as compared with that in vacuum.

\subsection{Global reactivity parameters}

The energies of frontier molecular orbitals ( $E_{\mathrm{HOMO}}$, $\left.E_{\mathrm{LUMO}}\right)$, energy band gap $\left(E_{\mathrm{HOMO}}-E_{\mathrm{LUMO}}\right)$, electronic chemical potential $(\mu)$, electronegativity $(\chi)$, global hardness $(\eta)$, global softness $(S)$, and electrophilicity indices $(\omega)$ have been listed in table 3, which were calculated on the basis of $E_{\mathrm{HOMO}}$ and $E_{\mathrm{LumO}}$, using the following equations. ${ }^{44}$

$$
\begin{gathered}
\mu=\frac{1}{2}\left(E_{\mathrm{LUMO}}+E_{\mathrm{HOMO}}\right) \\
\chi=-\mu=-\frac{1}{2}\left(E_{\mathrm{LUMO}}+E_{\mathrm{HOMO}}\right) \\
\eta=\frac{1}{2}\left(E_{\mathrm{LUMO}}-E_{\mathrm{HOMO}}\right) \\
S=\frac{1}{2 \eta} \\
\omega=\frac{\mu^{2}}{2 \eta}
\end{gathered}
$$

Table 3. Calculated energy values of "A" and "B" forms by B3LYP/6-311G(d,p) basis set.

\begin{tabular}{lcc}
\hline Chemical parameters & "A" form & "B” form \\
\hline$E_{\mathrm{HOMO}}(\mathrm{eV})$ & -6.0089 & -4.9426 \\
$E_{\mathrm{HOMO}-1}(\mathrm{eV})$ & -6.6745 & -6.2680 \\
$E_{\mathrm{LUMO}}(\mathrm{eV})$ & -2.4171 & -2.5464 \\
$E_{\mathrm{LUMO}+1}(\mathrm{eV})$ & -1.5896 & -1.4212 \\
$E_{\mathrm{HOMO}-\mathrm{LUMO}}$ gap $(\mathrm{eV})$ & 3.5918 & 2.3962 \\
Chemical potential $(\mu)$ & -4.2130 & -3.7445 \\
Global hardness $(\eta)$ & 1.7959 & 1.1981 \\
Global softness $(S)$ & 0.2784 & 0.4173 \\
Elecronegativity $(\chi)$ & 4.2130 & 3.7445 \\
Electrophilicity index $(\omega)$ & 4.9416 & 5.8515 \\
\hline
\end{tabular}


Electrophilicity index is one of the main quantum chemical descriptors in characterizing biological or toxicity activities of the molecules in the background of expansion of Quantitative Structure Activity Relationship (QSAR) parlance. ${ }^{45}$ The computed electrophilicity index of the title compound also describes the biological activity of drug-receptor interaction.

\subsection{Current-Voltage Characteristics of Linear Molecular Switches}

As illustrated in figure S7 (Supplementary Information), the molecular electronic device is divided into several regions: the right electrode, the central scattering region, and the left electrode. Figure 5 shows the calculated I-V characteristics of the system for the two forms at a bias up to $2 \mathrm{~V}$. It should be noted that at each bias, the current is determined self-consistently under the nonequilibrium condition. The switching action can be obviously seen in figure 5; the current of open ("B") form is greater than that of closed ("A") form at the same bias. It can be concluded that there is a switch from on (low resistance) state to the off (high
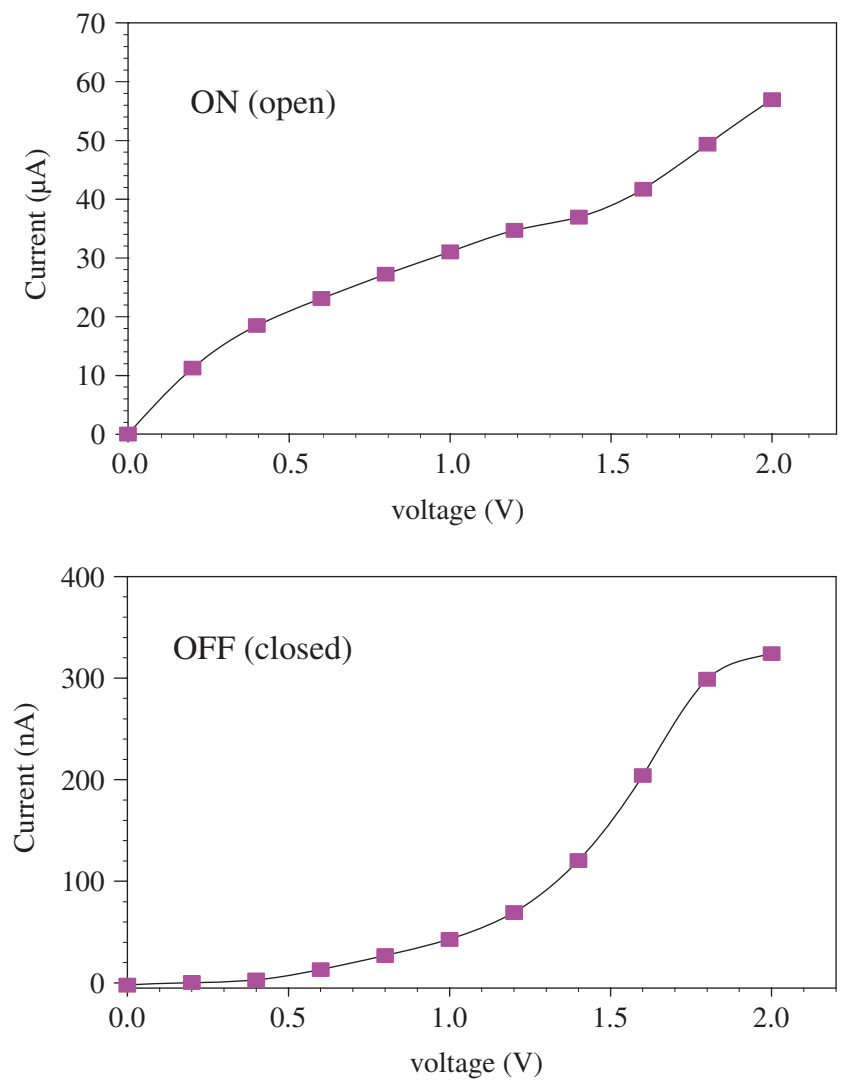

Figure 5. Calculated current-voltage $(I-V)$ characteristics for OFF and ON state structures, 'A' and 'B' forms, of the title compound. Note the different scales for the calculated currents in the $\mathrm{ON}$ and $\mathrm{OFF}$ state. resistance) state when the open form of the molecule changes to the closed form.

As shown in Landauer-Bütiker formula (Eq. 1), calculated values for the current for the system is dependent on the transmission spectra. The switching behavior of this compound can be perceived from the energy affiliation of zero bias transmission spectra, which are shown in figure S8 (Supplementary Information). In our computation, the average Fermi level is set as zero. From Eq. 1, we can expect that only electrons with energies within a range close the Fermi level $E_{F}$ contribute to the total current. Therefore, a good approximation of the range of the bias window, i.e., $[-\mathrm{V} / 2,+\mathrm{V} / 2]$ is adequate to analyze a finite section of the transmission spectrum.

It is clear in figure S8 that the transmission spectra of two isomers display tangibly disparate characteristics. When the system is with open form, the value of the transmission coefficient is smaller than that of closed form in the energy window of $[-2.0,2.0 \mathrm{eV}]$. Meanwhile, the HOMO and LUMO levels are -1.131 , $0.169 \mathrm{eV}$ for the open form and $-1.943,0.0 .063 \mathrm{eV}$ for the closed form. So, the HOMO-LUMO gap of the open form is smaller than that of the closed form. As a result, the absence of intense peaks in the window of $[-2.0,2.0 \mathrm{eV}]$ and larger HOMO-LUMO gap because of the molecular structure of closed form account for its low conductivity.

\section{Conclusions}

The novel compound the 2-([1,1'-biphenyl]-4-yl)2-methyl-6-(4-nitrophenyl)-4-phenyl-1,3 diazabicyclo [3.1.0]hex-3-ene was synthesized for the first time and characterized by IR, UV-Vis and NMR. The entire calculations for the title compound were done with a hybrid functional B3LYP at 6-311G(d,p) and basis set. The UV-Vis band in " $\mathrm{A}$ " (closed) form is observed at $310 \mathrm{~nm}$ (colorless), and for "B" (open) form at 310 and $415 \mathrm{~nm}$ (blue color) due to the formation of zwitterionic doubly charged imine ylide (open form). Also, molecular electrostatic potential map (MEP), frontier molecular orbital analysis (HOMO-LUMO), total density of state (TDOS) and reactivity descriptors were found and discussed.

Title compound has been proposed as a potential molecular switch for electronic device, in particular, a photoinduced molecular switch. This molecule has two stable conformations (closed and open) in its ground state, which makes it a promising component of molecular devices, and can be converted from one conformation to the other by photoexcitation. The open form 
("B") has a conductance considerably higher than that of the closed form ("A") at equilibrium (zero bias), enabling its use as a molecular switch with $\mathrm{ON}$ and $\mathrm{OFF}$ states.

\section{Supplementary Information (SI)}

All additional information pertaining to synthesis of the title compound, deconvoluted IR spectra (figure S1), correlation between the calculated and the experimental frequencies (figure S2), molecular electrostatic potential surface (figure S3), TDOS diagrams (figure S4), frontier molecular orbital (figure S5), calculated absorption and fluorescence spectra of open form (figure S6), schematic molecular junctions (figure S7), transmission spectra of the molecular switch at zero bias (figure S8), characterization of the geometry optimization of " $A$ " and " $B$ " forms (table S1), observed and calculated (scaled) selected frequencies $\left(\mathrm{cm}^{-1}\right)$ for " $A$ " and " $B$ " forms (table S2), experimental and theoretical ${ }^{1} \mathrm{H}$ NMR isotropic chemical shifts (table S3), are given in the Supporting Information, available at www.ias.ac. in/chemsci.

\section{Acknowledgments}

The authors are grateful to Dr. Mohammad Vakili (Ferdowsi University of Mashhad) for his kind assistance and help. Financial support of Damghan University is gratefully acknowledged.

\section{References}

1. Bousas-Laurent H and Durr H 2001 Pure Appl. Chem. 73639

2. Tighadouini S, Radi S, Toupet L, Sirajuddin M, Ben Hadda T, Akkurt M, Warad I, Mabkhot Y N and Ali S 2015 J. Chem. Sci. 1272211

3. Chen Y, Pang M L, Cheng K G, Wang Y, Han J, He Z J and Meng J B 2007 Tetrahedron 634319

4. Mahmoodi N O, Tabatabaeian K and Kiyani H 2012 Helv. Chim. Acta 95536

5. Mahmoodi N O and Kiyani H 2004 Bull. Korean Chem. Soc. 251417

6. Kamidate T, Yamaguchi K and Segawa T 1989 Anal. Sci. 5429

7. Ucucu U, Karaburun N G and Isikdag I 2001 IL Farmaco 56285

8. Eaton D F 1991 Science 253281

9. Becker F F and Banik B K 1998 Bioorg. Med. Chem. 50 2877

10. Joachim C, Gimzewski J K and Aviram A 2000 Nature 408541

11. Poulsen K M and Bjørnholm T 2009 Nat. Nanotechnol. 4551

12. Galperin M and Nitzan A 2012 Phys. Chem. Chem. Phys. 149421
13. Vakili M, Tayyari S F, Kanaani A, Nekoei A R, Salemi S, Miremad H, Berenji A R and Sammelson R E J. Mol. Struct. 99899

14. Vakili M, Nekoei A R, Tayyari S F, Kanaani A and Sanatid N 2012 J. Mol. Struct. 1021102

15. Kanaani A, Ajloo D, Kiyani H and Farahani M $2014 J$. Mol. Struct. 106330

16. Kanaani A, Ajloo D, Ghasemian H, Kiyani H, Vakili M and Mosallanezhad A 2015 Struct. Chem. 261095

17. Kiyani H, Kanaani A, Ajloo D, Ghorbani F and Vakili M 2014 Res. Chem. Intermed. 417739

18. Frisch M J, Trucks G W, Schlegel H B, Scuseria G E, Robb M A, Cheeseman J R, Scalmani G, Barone V, Mennucci B, Petersson G A, Nakatsuji H, Caricato M, Li X, Hratchian H P, Izmaylov A F, Bloino J, Zheng G, Sonnenberg J L, Hada M, Ehara M, Toyota K, Fukuda R, Hasegawa J, Ishida M, Nakajima T, Honda Y, Kitao O, Nakai H, Vreven T, Montgomery J A Jr, Peralta J E, Ogliaro F, Bearpark M, Heyd J J, Brothers E, Kudin K N, Staroverov V N, Keith T, Kobayashi R, Normand J, Raghavachari K, Rendell A, Burant J C, Iyengar S S, Tomasi J, Cossi M, Rega N, Millam J M, Klene M, Knox J E, Cross J B, Bakken V, Adamo C, Jaramillo J, Gomperts R, Stratmann RE, Yazyev O, Austin A J, Cammi R, Pomelli C, Ochterski J W, Martin R L, Morokuma K, Zakrzewski V G, Voth G A, Salvador P, Dannenberg J J, Dapprich S, Daniels A D, Farkas O, Foresman J B, Ortiz J V, Cioslowski J, Fox D J 2004 Gaussian 03, Revision E.01 (Gaussian, Inc. : Wallingford)

19. Foresman J B 2000 In Exploring Chemistry with Electronic Structure Methods: A Guide to Using Gaussian (Pittsburg: Gaussian Inc)

20. Wolinski K, Hinton J F and Pulay P 1990 J. Am. Chem. Soc. 1128251

21. Casida M E, Casida K C and Salahub D R 1998 Int. J. Quantum Chem. 70933

22. Brandbyge M, Mozos J L, Ordejon P, Taylor J and Stokbro K 2002 Phys. Rev. B 65165401

23. Cao Y, Ge Q, Dyer D and Wang L 2003 J. Phys. Chem. B 1073803

24. Soler J M, Artacho E, Gale J D, García A, Junquera J, Ordejón P and Sánchez-Portal D J 2002 J. Phys.: Condens. Matter 142745

25. Troullier N and Martins J L 1991 Phys. Rev. B 431993

26. Perdew J P, Burke K and Ernzerhof M 1996 Phys. Rev. Lett. 773865

27. Zhang G P, Hu G C, Li Z L and Wang C K 2012 J. Phys. Chem. C 1163773

28. Sett P, Chattopadhyay S and Mallick P K 2000 Spectrochim. Acta A $\mathbf{5 6} 855$

29. Dhanabal T, Amirthaganesan G, Dhandapani M and Das S K 2012 J. Chem. Sci. 124951

30. Sundaraganesan N, Ayyappan S, Umamaheswari H and Joshua B D 2007 Spectrochim. Acta A 6617

31. Jensen J O, Banerjee A, Merrow C N, Zeroka D and Lochner J M 2000 J. Mol. Struct. (THEOCHEM) 531 323

32. Kumar V and Balachandran V 2005 Spectrochim. Acta A 611811

33. Roeges N P G 1994 In A Guide to the Complete Interpretation of Infrared Spectra of Organic Structures (New York: Wiley) 
34. Suryanarayana V, Kumar A P, Rao G R and Panday G C 1992 Spectrochim. Acta A 481481

35. Shinde Y, Sproules S, Kathawate L, Pal S, Konkimalla V B and Salunke-Gawali S 2014 J. Chem. Sci. 126213

36. Melardi M R, Shamsi Mogoll F B, Baball Sajirani A, Attar Gharamaleki J, Notash B and Rofouei M K 2015 J. Chem. Sci. 1272171

37. Gunasekaran S, Kumaresan S, Arunbalaji R, Anand G and Srinivasan S 2008 J. Chem. Sci. 120315

38. Sajan D, Jothy V B, Kurulvilla T and Joe I H 2010 J. Chem. Sci. 22511

39. El-Azhary A A 1999 Spectrochim. Acta A 552437
40. Gunasekaran S, Balaji R A, Kumaresan S, Anand G and Srinivasan S 2008 Can. J. Anal. Sci. Spectros. 53149

41. N O'Boyle N M, Tenderholt A L and Langner K M 2008 J. Comp. Chem. 29839

42. Fukui K 1982 Science $\mathbf{2 1 8} 747$

43. Kumar S, Sharma R, Kumar S and Nitika 2014 Chem. Sci. Trans. 3919

44. Padmanabhan J, Parthasarathi R, Subramanian V and Chattaraj P K 2007 J. Phys. Chem. A 1111358

45. Garrido A P, Helguera A M, Guillén A A, Cordeirom M N D S and Escudero A G 2009 Bioorgan. Med. Chem. 17896 